\title{
Acoustomagnetoelectric effect in two-dimensional materials: Geometric resonances and Weiss oscillations
}

\author{
I. G. Savenko, ${ }^{1,2}$ A. V. Kalameitsev,$^{2}$ L. G. Mourokh, ${ }^{3}$ and V. M. Kovalev ${ }^{2,4}$ \\ ${ }^{1}$ Center for Theoretical Physics of Complex Systems, \\ Institute for Basic Science (IBS), Daejeon 34126, Korea \\ ${ }^{2}$ Rzhanov Institute of Semiconductor Physics, Siberian Branch, \\ Russian Academy of Sciences, Novosibirsk, 630090 Russia \\ ${ }^{3}$ Physics Department, Queens College of the City University of New York, Flushing, NY 11367, USA \\ ${ }^{4}$ Novosibirsk State Technical University, Novosibirsk, 630072 Russia
}

(Dated: April 29, 2020)

\begin{abstract}
We study electron transport in two-dimensional materials with parabolic and linear (graphene) dispersions of the carriers in the presence of surface acoustic waves and an external magnetic field using semiclassical Boltzmann equations approach. We observe an oscillatory behavior of both the longitudinal and Hall electric currents as functions of the surface acoustic wave frequency at a fixed magnetic field and as functions of the inverse magnetic field at a fixed frequency of the acoustic wave. We explain the former by the phenomenon of geometric resonances, while we relate the latter to the Weiss-like oscillations in the presence of the dynamic superlattice created by the acoustic wave. Thus we demonstrate the dual nature of the acoustomagnetoelectric effect in two-dimensional electron gas.
\end{abstract}

\section{INTRODUCTION}

Two-dimensional (2D) electronic systems have attracted great interest of researchers for several recent decades. Initially, two-dimensional electron gas (2DEG) was realized in the inversion layer at the interface of two semiconductors with different bandgaps [1. Subsequently, other structures based on graphene [2, 3] and metal dichalcogenides 4 were created. One of the primary motivations to design a system containing $2 \mathrm{DEG}$ is that it represents an ideal platform for the studies of magnetotransport which led to the observations of quantum Hall [5] and fractional quantum Hall [6, 7] effects.

Other prominent phenomenology is related to magneto-oscillations of various types. Some of them are connected to quantum effects at relatively high magnetic fields when the Landau quantization causes the Shubnikov-de Haas effect and associated oscillations 8. Quantum interference between trajectories gives rise to Aharonov-Bohm oscillations in high-mobility GaAs/AlGaAs heterostructures 9. On the other hand, semiclassical effects, which can be observed at smaller fields or higher temperatures, are Weiss [10] and BrownZak (BZ) oscillations [11, 12. The former arises due to the commensurability between the cyclotron orbit and the spatial period in the structure, while the latter is related to the commensurability between the magnetic flux through the unit cell area and the magnetic flux quantum. Subsequent Landau quantization of the BZ minibands leads to the fractal Hofstadter Butterfly (HB) spectrum [13. Since the area of the crystal unit cell is small, it is necessary to apply extremely high fields to detect the associated phenomenology. However, in bilayer graphene or in monolayer graphene placed on top of a hexagonal boron nitride, additional moir patterns appear, which allows to observe both HB [14-16] and BZ [17] oscillations.
There also exist other types of oscillations in 2D systems. One of them is called the geometric resonances (GRs). Originally, they revealed themselves in the spectra of electromagnetic power absorption coefficient of plasmas in gases and solids in the presence of a uniform magnetic field [18, 19. The GRs appear as a multi-peak structure at frequencies $\omega=l \omega_{c}$, where $l$ is integer, in addition to the conventional cyclotron (or magnetoplasmon) peak at the cyclotron frequency $\omega_{c}=e B / m$ (or $l=1$ ) with $e$ and $m$ being the electron charge and mass, $B$ is the strength of the external magnetic field. The GRs in 2D systems have been studied theoretically [20, 21] and reported experimentally 22 in samples made of various materials, such as Si and AlGaAs alloys.

In this paper, we examine magnetotransport phenomena in a 2DEG in the presence of surface acoustic waves (SAWs). These waves are usually produced by the interdigital transducers (IDTs) - metallic gates patterned on top of piezoelectric materials. The spacing of the gates, or pitch, determines the wavelength of the SAW [23]. When the radio-frequency (rf) signal is applied to ITDs, there emerges a SAW with such a wavelength that its product with the rf frequency equals to the sound velocity of the material. Corresponding piezoelectric field modulates both the electron density and velocity of the charge carriers. Accordingly, the electric current density, which is the product of these two parameters, acquires a constant component, called the acoustoelectric current. It can also be explained as a result of SAW drag of the charge carriers in the direction of the SAW wave vector 24. The information obtained by measurements of the SAW-induced effects is complementary to conventional transport experiments, facilitating a frequent use of SAWs in the studies of low-dimensional electronic structures [25], including graphene monolayers [26 28], topological insulators [29], and other thin films [30. Besides, SAWs-related methods can also be applied to the exciton transport 31 33. 
The response of an electron-exposed-to-SAWs system to an external magnetic field was also examined in Refs. [34 37, although these studies were focused on the quantum regime with established Landau levels. A region of smaller fields was considered in Refs. [38, 39] but the manifestations of Weiss oscillations were only predicted for the first-order effects, such as the SAW absorption and the velocity shifts. The longitudinal component of the acoustoelectric current was discussed in Ref. 40]. Here, we extend this analysis to the Hall component and also examine the peculiarities appearing in the case of the linear dispersion of graphene.

The acoustoelectric current is the second-order effect with respect to the SAW-induced electric field. Consequently, it is related to a third-order conductivity tensor [41, 42. This tensor couples components of the drag current to the components of the SAW piezoelectric field as $j_{\alpha}=\chi_{\alpha \beta \gamma} E_{\beta} E_{\gamma}$, where $\alpha, \beta, \gamma=x, y, z$, similar to the photovoltaic effect [43. As the SAW frequency is much smaller than the frequencies of the optical fields reported in Refs. 20 22, GRs can be expected at much smaller magnetic fields, at which a semiclassical approach based on the Boltzmann equations is appropriate for our studies.

We calculate both the longitudinal and Hall current densities as functions of the SAW frequency and the magnetic field for two possible cases of (i) the parabolic dispersion (for the 2DEG of an interface inversion layer or of a transition metal dichalcogenide) and (ii) the linear dispersion of graphene, and we obtain an oscillatory behavior of these dependencies. We analyze these oscillations and argue that in the case of the SAW drag, GRs and Weiss oscillations represent the same phenomenon; although originally GRs are related to the optical fields with no spatial periodicity and Weiss oscillations are usually connected with a static embedded superlattice. SAWs thus provide a dynamical superlattice merging the GRs and Weiss oscillations phenomena and making both interpretations possible.

\section{THEORETICAL FRAMEWORK}

We start with the Boltzmann equation for the electron distribution function $f$, when the system is subject to both the piezoelectric field of the SAW and the external uniform magnetic field perpendicular to the 2D layer. In the case of the parabolic electron dispersion, the Boltzmann equation has the form

$$
\begin{array}{r}
{\left[\frac{\partial}{\partial t}+\mathbf{v} \frac{\partial}{\partial \mathbf{r}}+e\left(\mathbf{E}(\mathbf{r}, t)+\mathbf{E}^{i}(\mathbf{r}, t)\right)\right.} \\
\left.+e[\mathbf{v} \times \mathbf{B}] \frac{\partial}{\partial \mathbf{p}}\right] f=-\frac{f-\langle f\rangle}{\tau},
\end{array}
$$

where $\mathbf{v}=\mathbf{p} / m$ is a velocity of a particle (thus the energy spectrum is given by $\left.\varepsilon_{\mathbf{p}}=\mathbf{p}^{2} / 2 m\right), \mathbf{r}$ is the coordinate, and $\tau$ is an effective electron scattering time. SAWs produce the in-plane component of a piezoelectric field $\mathbf{E}(\mathbf{r}, t)$ directed along the SAW wave vector $\mathbf{k}, \mathbf{E}(\mathbf{r}, t) \| \mathbf{k}$. $\mathbf{E}^{i}(\mathbf{r}, t)$ is the induced field due to the spatial modulation of $2 \mathrm{D}$ electron density in SAW field, which can be found from the solution of the Maxwell's equation. $\langle f\rangle$ is a quasi-equilibrium electron distribution function in the SAW reference frame. This function depends on time and coordinates via the chemical potential $\mu(\mathbf{r}, t)$, which determines the electron density $n(\mathbf{r}, t)$ in slow-varying SAW field.

To find the acoustoelectric current, we expand the electron density and the distribution functions up to the second-order with respect to the total electric field $\tilde{\mathbf{E}}(\mathbf{r}, t)=\mathbf{E}(\mathbf{r}, t)+\mathbf{E}^{i}(\mathbf{r}, t)$. In particular, $f(\mathbf{r}, t)=f_{0}+f_{1}(\mathbf{r}, t)+f_{2}(\mathbf{r}, t)+o\left(f_{3}\right)$, where $f_{0}$ is the equilibrium electron distribution function. The first-order correction to $f_{0}$ is $f_{1}(\mathbf{r}, t)=$ $\left[f_{1} \exp (i \mathbf{k} \cdot \mathbf{r}-i \omega t)+f_{1}^{*} \exp (-i \mathbf{k} \cdot \mathbf{r}+i \omega t)\right] / 2, \quad$ where $\omega=s|\mathbf{k}|=s k$, with $s$ being the sound velocity.

The time-independent acoustoelectric current can be determined from the stationary second-order correction to the electron distribution function $f_{2}$ with respect to the SAW field $\mathbf{E}(\mathbf{r}, t)$, as

$$
\mathbf{j}=e \int \frac{d \mathbf{p}}{(2 \pi \hbar)^{2}} \mathbf{v} f_{2} \text {. }
$$

Furthermore, we consider 2DEG to be highly degenerate, thus all the parameters are taken at the Fermi energy. The $x$-axis is chosen along the direction of the SAW propagation. After the calculations detailed in Appendix A and Appendix B, Sec. a, we obtain the longitudinal and Hall acoustoelectric currents in the parabolic electron dispersion case, as

$$
\begin{aligned}
\left(\begin{array}{c}
j_{x} \\
j_{y}
\end{array}\right) & =\frac{1}{e n v_{F}}\left|\frac{\sigma_{0} E_{0}}{g(k, \omega)}\right|^{2} \frac{1}{\beta_{F}^{2}\left(1+\omega_{c}^{2} \tau^{2}\right)} \\
& \times \operatorname{Re} \sum_{l} \frac{J_{l}\left(\beta_{F}\right)}{1-i\left(\omega-l \omega_{c}\right) \tau}\left[l+\frac{k a_{0}}{\omega_{c} \tau} \frac{\sigma_{x x}}{\varepsilon_{0}\left(s-R_{x}\right)}\right] \\
& \times\left(\begin{array}{c}
\gamma(l+1) J_{l+1}\left(\beta_{F}\right)+\gamma^{*}(l-1) J_{l-1}\left(\beta_{F}\right) \\
i \gamma(l+1) J_{l+1}\left(\beta_{F}\right)-i \gamma^{*}(l-1) J_{l-1}\left(\beta_{F}\right)
\end{array}\right),
\end{aligned}
$$

where $\sigma_{0}=e^{2} n \tau / m$ is a static Drude conductivity, $E_{0}$ is the amplitude of the (external) piezoelectric field, and $J_{l}\left(\beta_{F}\right)$ are the ordinary Bessel functions with $\beta_{F}=$ $k v_{F} / \omega_{c}$. We have also introduced two auxiliary parameters, $\gamma=1+i \omega_{c} \tau$ and $a_{0}=2 \pi \hbar^{2} \varepsilon_{0} / m e^{2}$. The $x x$-component of the conductivity tensor $\sigma_{x x}$ and $x$ component of the generalized diffusion coefficient $R_{x}$ are given by

$$
\sigma_{x x}=\frac{2 \sigma_{0}}{\beta_{F}^{2}} \sum_{l} \frac{l^{2} J_{l}^{2}\left(\beta_{F}\right)}{1-i\left(\omega-l \omega_{c}\right) \tau}
$$

and

$$
R_{x}=\frac{\omega_{c}}{k} \sum_{l} \frac{l J_{l}^{2}\left(\beta_{F}\right)}{1-i\left(\omega-l \omega_{c}\right) \tau}
$$



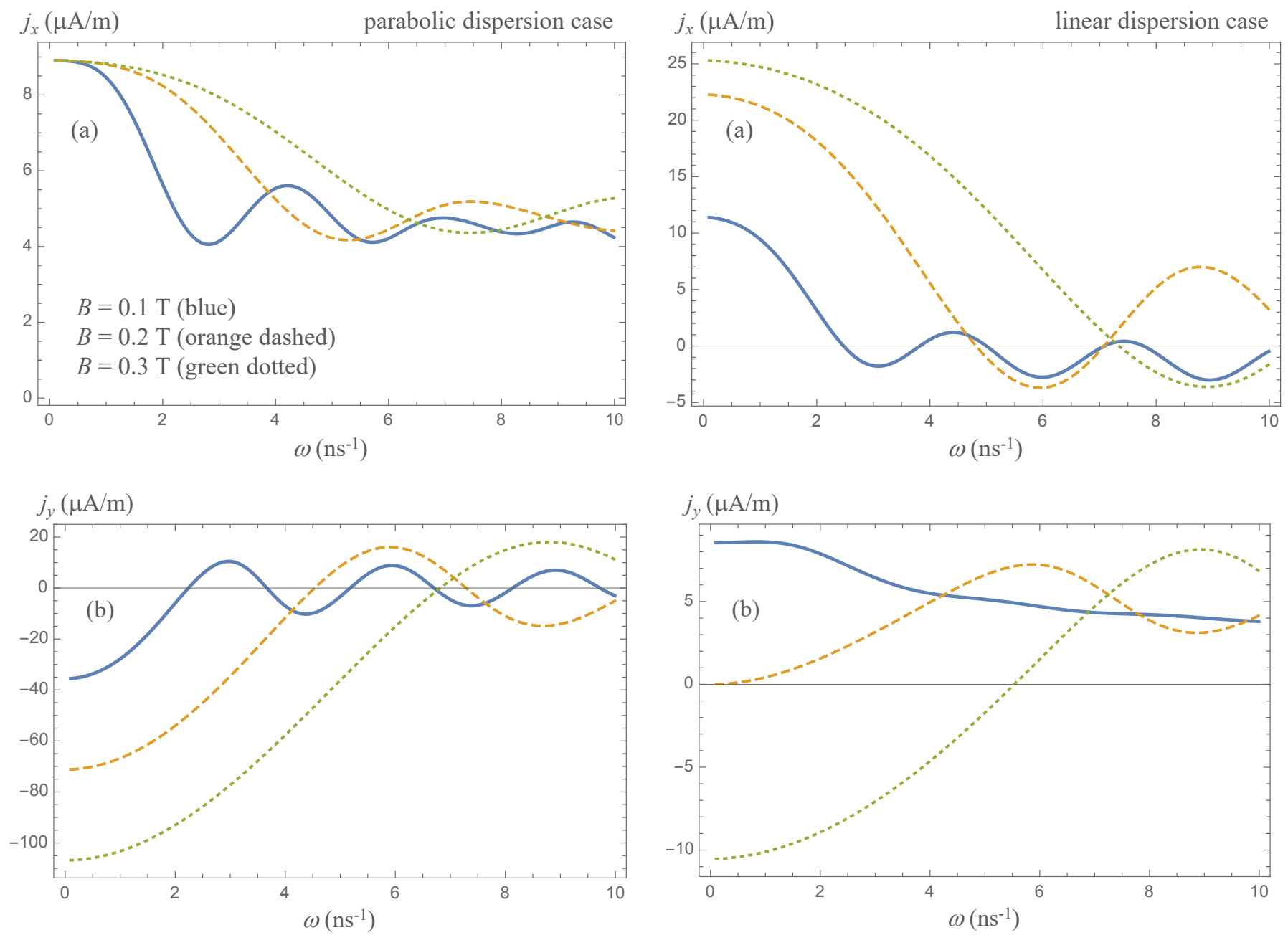

FIG. 1: (Color online) Electric current densities as functions of the SAW frequency for the parabolic dispersion case. (a) Longitudinal drag ( $x$-component) and (b) Hall drag (ycomponent). Different colors correspond to different values of the applied magnetic field $B$, specified in panel (a).

respectively, where

$$
g(k, \omega)=1+i \frac{1}{\epsilon_{0}\left(\epsilon_{d}+1\right)} \frac{\sigma_{x x}}{\left(s-R_{x}\right)}
$$

is the dielectric function of 2DEG, $\epsilon_{0}$ is the dielectric permittivity of free space, and $\epsilon_{d}$ is the dielectric constant of the substrate. The function of Eq. (6) describes the screening of SAW piezoelectric field by the mobile electrons of $2 \mathrm{D}$ system.

In the case of linear electron spectrum, $\varepsilon_{\mathbf{p}}=v_{0} p$, the Boltzmann equation remains almost the same as Eq. (1) with the number of changes. First, velocity $\mathbf{v}$ is replaced by $v_{0} \mathbf{p} / p$. Second, even for short-range impurities, the scattering times of the first and second harmonics of electron distribution function become energy-dependent, as $\tau_{1}(p) \equiv \tau_{1}\left(\varepsilon_{\mathbf{p}}\right)=\tau \varepsilon_{F} / \varepsilon_{\mathbf{p}}$ for the first harmonics, and $\tau_{2}(p)=\tau_{1}(p) / 2$ for the second harmonics [4]. Third, the

FIG. 2: (Color online) Electric current densities as functions of the SAW frequency for the liner dispersion case. (a) Longitudinal drag ( $x$-component) and (b) Hall drag ( $y$-component). Different colors correspond to different values of the applied magnetic field $B$, specified in Fig. 1(a).

effective cyclotron frequency in the semiclassical limit is given by $\omega_{c}(p)=e B v_{0} / p=e B v_{0}^{2} / \varepsilon_{p}$, 45, 46].

Performing the calculations (see Appendix B, Sec. b), we obtain the longitudinal and Hall acoustoelectric current densities in the linear electron dispersion case, as

$$
\begin{aligned}
& \left(\begin{array}{l}
j_{x} \\
j_{y}
\end{array}\right)=\frac{1}{2 e n v_{0}}\left|\frac{\sigma_{g} E_{0}}{g(k, \omega)}\right|^{2}\left(\frac{1 / \beta_{p_{F}}}{1+\omega_{c}^{2}\left(p_{F}\right) \tau_{2}^{2}\left(p_{F}\right)}\right)^{2}(7) \\
& \times \operatorname{Re} \sum_{l} \frac{J_{l}\left(\beta_{p_{F}}\right)}{1-i\left[\omega-l \omega_{c}\left(p_{F}\right)\right] \tau_{1}\left(p_{F}\right)} \\
& \times\left[l+\frac{k a_{g}}{\omega_{c}\left(p_{F}\right) \tau_{1}\left(p_{F}\right)} \frac{\sigma_{x x}}{\varepsilon_{0}\left(s-R_{x}\right)}\right] \\
& \times\left(\begin{array}{c}
-i \bar{\gamma}^{2}(l+1) J_{l+1}\left(\beta_{p_{F}}\right)+i \bar{\gamma}^{* 2}(l-1) J_{l-1}\left(\beta_{p_{F}}\right) \\
\bar{\gamma}^{2}(l+1) J_{l+1}\left(\beta_{p_{F}}\right)+\bar{\gamma}^{* 2}(l-1) J_{l-1}\left(\beta_{p_{F}}\right)
\end{array}\right),
\end{aligned}
$$

where $\sigma_{g}=e^{2} n v_{0} \tau_{1}\left(p_{F}\right) / p_{F}$ is a static Drude conduc- 
tivity in graphene and all the momentum-dependent quantities are taken at $p=p_{F}$. In particular, $\bar{\gamma}=$ $1+i \omega_{c}\left(p_{F}\right) \tau_{2}\left(p_{F}\right)$ and $a_{g}=2 \pi \hbar^{2} \varepsilon_{0} v_{0} / e^{2} p_{F}$. In this case, the $x x$-component of the conductivity tensor and $x$-component of the generalized diffusion coefficient have the forms

$$
\sigma_{x x}=\frac{2 \sigma_{g}}{\beta_{p_{F}}^{2}} \sum_{l} \frac{l^{2} J_{l}^{2}\left(\beta_{p_{F}}\right)}{1-i\left[\omega-l \omega_{c}\left(p_{F}\right)\right] \tau_{1}\left(p_{F}\right)}
$$

and

$$
R_{x}=\frac{\omega_{c}\left(p_{F}\right)}{k} \sum_{l} \frac{l J_{l}^{2}\left(\beta_{p_{F}}\right)}{1-i\left[\omega-l \omega_{c}\left(p_{F}\right)\right] \tau_{1}\left(p_{F}\right)},
$$

respectively. We immediately see several similarities and differences between Eqs. (8), (9) and Eqs. (4), (5), which we discuss below.

\section{RESULTS AND DISCUSSION}

First of all, we want to stress that the argument $\beta_{F}$ of the Bessel functions in Eqs. (3)-(5) and Eqs. (7)-(9) is of special interest. On one hand, it can be expressed in terms of the ratio of frequencies, as $\beta_{F}=\omega v_{F} / \omega_{c} s$ (in the parabolic case), resembling the GRs. On the other hand, $\beta_{F}$ represents the ratio of the space scales, as $\beta_{F}=$ $k r_{c}=2 \pi r_{c} / \lambda$, where $r_{c}$ is the cyclotron radius, which is very similar to Weiss oscillations.

To evaluate the electric current densities given by Eqs. (3) and (7), we use the following set of parameters: $E_{0}=10 \mathrm{kV} / \mathrm{m} ; n=5 \cdot 10^{12} \mathrm{~cm}^{-2}$, which is an experimentally achievable value [46] $m=0.44 m_{0}$, where $m_{0}$ is a free electron mass, and we choose $\mathrm{MoS}_{2}$ as a material with the parabolic spectrum; and $\tau=10^{-10} \mathrm{~s}$, which corresponds to moderately clean samples. The parameters of the piezoelectric substrate are $\epsilon_{d}=50$ and $s=3.5 \cdot 10^{3} \mathrm{~m} / \mathrm{s}$, taken for $\mathrm{LiNbO}_{3}$. For graphene, $v_{0}=$ $10^{8} \mathrm{~cm} / \mathrm{s}$ and $\tau_{1}=\mu_{e} p_{F} / e v_{0}$, where $\mu_{e}=10^{4} \mathrm{~cm}^{2} / \mathrm{V} \cdot \mathrm{s}$ is the electron mobility 47, 48.

Figures 1 and 2 show (a) longitudinal and (b) Hall components of the drag current density as functions of the SAW frequency $\omega$ for the cases of the parabolic and linear dispersions of mobile carriers, respectively, at various values of the external magnetic field. It is evident from these figures that both components exhibit oscillations, with each maximum approximately corresponding to the geometric resonance $\omega=l \omega_{c}$. As expected, for relatively small SAW frequencies and the cyclotron frequency increasing with $B$, the GRs are pronounced at magnetic fields smaller than $1 \mathrm{~T}$. At higher fields, the functions are monotonic with no GRs-related oscillations.

The dependencies of the current density components on the inverse magnetic field are demonstrated in Figs. 3 and 4 for the parabolic and linear dispersion cases, respectively. One can see almost perfect oscillations superimposed onto the monotonic decay to the zero field. They are more pronounced for the parabolic dispersion of electrons. This result can be understood as Weiss oscillations in the presence of the spatial periodic structure of the SAW.

Another prominent feature, which we observe in the plots, is the change of the sign of the Hall current density in both the parabolic and linear dispersion cases, and the longitudinal current density in the graphene case. The Hall current vanishes at zero fields and monotonically increases with the increase of $B$. In the presence of SAW-induced oscillations of relatively high magnitude, the current density at small field can achieve negative values at minima. The longitudinal component of acoustoelectric current is non-zero even without a magnetic field. For the parabolic electron dispersion, the magnitude of the oscillations is not sufficiently large to reach negative values of the current density, while for graphene it can occur since the oscillations are more pronounced.

It should be noted that a similar effect of the sign change was also observed in the photon drag in graphene [4], where it was attributed to the energy dependence of the electron scattering time. We believe that the same phenomenology leads to the change of the sign of the acoustoelectric current. We also want to emphasize that the predicted oscillating behavior of the acoustoelectric current occurs at the range of field satisfying $\hbar \omega_{c} \ll E_{F}$, where $E_{F}$ is the Fermi energy, validating the usage of the semiclassical approach.

\section{CONCLUSIONS}

To summarize, we have examined acoustoelectric current in a $2 \mathrm{DEG}$ in the presence of an external magnetic field in two physical systems. First, we have considered 2DEG in which the electron energy is proportional to its momentum squared (parabolic dispersion case). In particular, such situation occurs at the interface of two semiconductors with different band gaps and in transition metal dichalcogenides. Second, we have studied 2DEG in graphene, where the energy is proportional to the first power of momentum (linear dispersion case).

The piezoelectric field created by the SAW modulates both the electron density and electron velocity, resulting in a permanent electric current as a second-order response of the system. Using the semiclassical Boltzmann equations approach, we have calculated and studied both the longitudinal and Hall current densities. For a fixed magnetic field, both the components of the acoustoelectric current exhibit oscillations as functions of the SAW frequency. We have shown that the Hall component changes its sign in both cases of parabolic and linear dispersions, while the change of sign of the longitudinal component occurs in graphene only. For a fixed SAW frequency, the acoustoelectric current oscillates as a function of the inverse magnetic field.

Mathematically, the oscillations are originating from the presence of the (ordinary) Bessel functions in the equations. The argument of Bessel functions can be rep- 

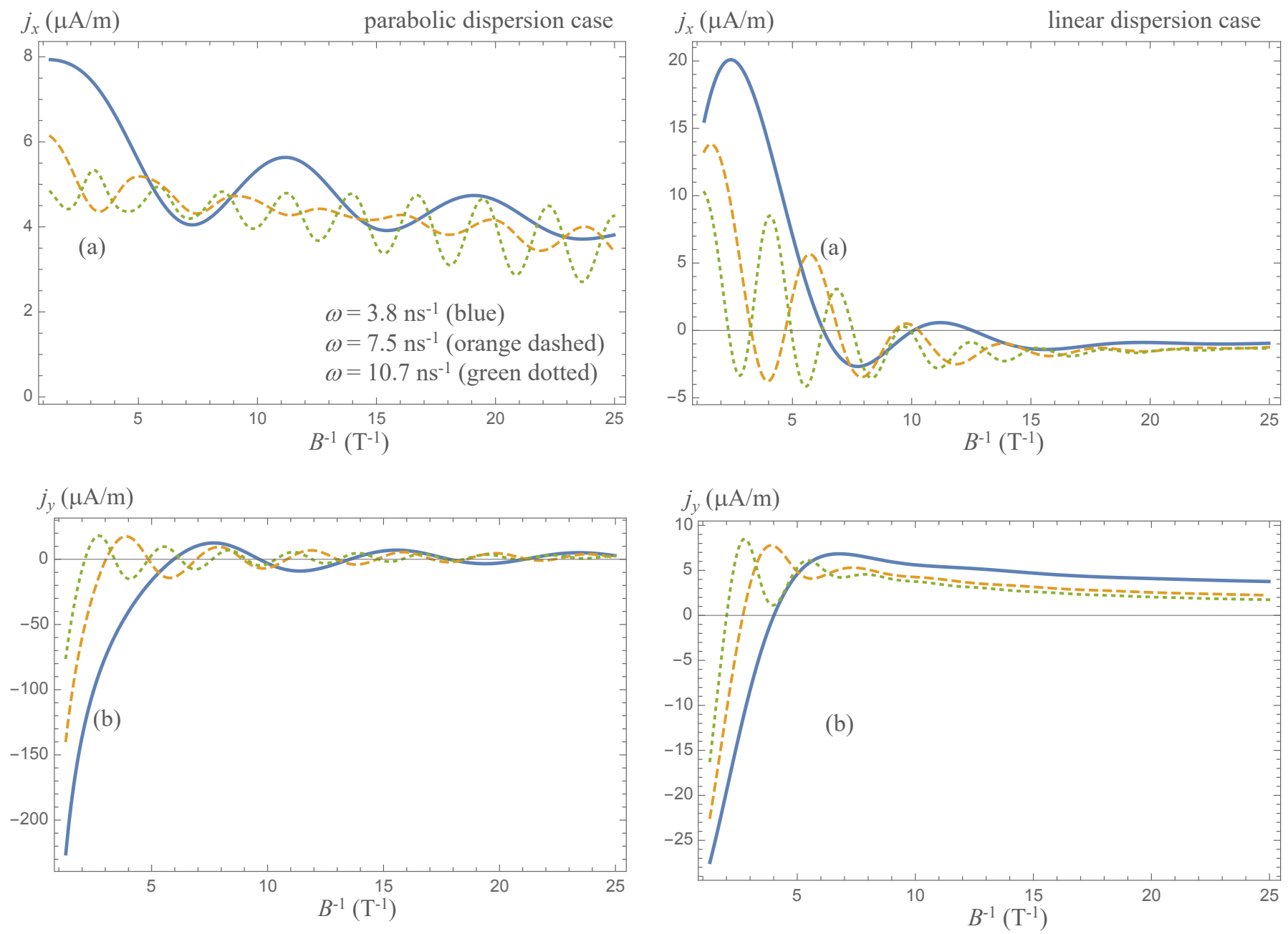

FIG. 3: (Color online) Components of electric current density as functions of inverse magnetic field in the case of parabolic dispersion for the frequencies specified in panel (a).

resented as a ratio of the SAW and cyclotron frequencies or as a ratio of the cyclotron radius and the SAW wavelength. The former is conventionally used to describe optical geometric resonances, while the latter appears in Weiss oscillations of magnetoresistance in the presence of an embedded static superlattice. In the case of SAWs, both interpretations of this phenomenology become possible, since these two effects merge.

\section{Acknowledgments}

We thank M. Malishava for useful discussions. IGS acknowledges the support by the Institute for Basic Science in Korea (Project No. IBS-R024-D1). AVK and VMK were supported by the Russian Foundation for Basic Research (Project No. 19-42-540011). LGM acknowledges the partial support by AFOSR, Award No. FA9550-161-0279.

\section{Appendix A: The first-order correction to the \\ electron distribution function}

FIG. 4: (Color online) Components of electric current density as functions of inverse magnetic field in the case of linear dispersion for the frequencies specified in Fig. 3(a).

The first-order corrections to the equilibrium electron distribution function and the electron density, $f_{1}(\mathbf{r}, t)$ and $n_{1}(\mathbf{r}, t)$, satisfy the Boltzmann equation [derived from Eq. (1)],

$$
\begin{gathered}
\left(\frac{1}{\tau}+i \mathbf{k} \cdot \mathbf{v}-i \omega+e[\mathbf{v} \times \mathbf{B}] \cdot \frac{\partial}{\partial \mathbf{p}}\right) f_{1}= \\
=-e\left(\mathbf{E}+\mathbf{E}^{i}\right) \frac{\partial f_{0}}{\partial \mathbf{p}}+\frac{n_{1}}{\tau} \frac{\partial f_{0}}{\partial n}
\end{gathered}
$$


To find this equation, we used the expansions

$$
\begin{gathered}
n(\mathbf{r}, t)=n+n_{1}(\mathbf{r}, t)+n_{2}(\mathbf{r}, t)+o\left(n_{3}\right), \\
f(\mathbf{r}, t)=f_{0}+f_{1}(\mathbf{r}, t)+f_{2}(\mathbf{r}, t)+o\left(f_{3}\right), \\
\langle f(\mathbf{r}, t)\rangle=f_{0}+\left[n_{1}(\mathbf{r}, t)+n_{2}(\mathbf{r}, t)+\ldots\right] \frac{\partial f_{0}}{\partial n}+ \\
+\frac{\left[n_{1}(\mathbf{r}, t)+n_{2}(\mathbf{r}, t)+\ldots\right]^{2}}{2} \frac{\partial^{2} f_{0}}{\partial n^{2}} .
\end{gathered}
$$

Following the approach described in [50], we switch to the polar system of coordinates, in which Eq. A1 reads

$$
\begin{gathered}
\left(\frac{1}{\tau}-i \omega+i k v \cos \phi-\omega_{c} \frac{\partial}{\partial \phi}\right) f_{1}(p, \phi) \\
=-e \tilde{E}_{0} v \cos \phi \frac{\partial f_{0}}{\partial \varepsilon_{p}}+\frac{n_{1}}{\tau} \frac{\partial f_{0}}{\partial n},
\end{gathered}
$$

where we have accounted for the fact that $\varepsilon_{\mathbf{p}}=\varepsilon_{p}$, where $p=|\mathbf{p}|$, and $\partial_{\mathbf{p}} f_{0}=\left(\partial_{\mathbf{p}} \varepsilon_{p}\right)\left(\partial_{\varepsilon_{p}} f_{0}\right)=\mathbf{v} \partial_{\varepsilon_{p}} f_{0}$ with $\partial_{B} A=$ $\partial A / \partial B$. We have also chosen the direction of $\mathbf{E}_{0}$ along the x-axis. Then $\tilde{\mathbf{E}}_{0}=\mathbf{E}_{0}+\mathbf{E}_{0}^{i}$ is also directed along the X-axis (since $\mathbf{E}_{0}$ and $\mathbf{k}$ are collinear). Then $\tilde{\mathbf{E}}_{0} \cdot \mathbf{v}=$ $\tilde{E}_{0} v \cos \phi$ and $k_{x}=k=\omega / s$.

Eq. A3 can be rewritten in the form

$$
\frac{\partial f_{1}}{\partial \phi}+i(\alpha-\beta \cos \phi)=Q(\phi)
$$

where

$$
\begin{array}{r}
\alpha=\frac{\omega+i / \tau}{\omega_{c}}, \quad \beta=\frac{k v}{\omega_{c}}=\frac{\omega v}{s \omega_{c}}, \\
Q(\varphi)=\left(\frac{e \tilde{E}_{0} v}{\omega_{c}} \cos \phi+\frac{n_{1}}{\omega_{c} \tau} \frac{\partial \mu}{\partial n}\right) \frac{\partial f_{0}}{\partial \varepsilon_{p}}
\end{array}
$$

which (all) evidently represent functions of frequency. In Eq. (A5), we used the relation $\partial_{n} f_{0}=\left(\partial_{\mu} f_{0}\right)\left(\partial_{n} \mu\right)$ and $\partial_{\mu} f_{0}=-\partial_{\varepsilon_{p}} f_{0}$, which holds for the Fermi distribution function.

From Eqs. A4 - A5 we find

$$
f_{1}(p, \phi)=-e^{i \beta \sin \phi} \int_{0}^{\infty} d \psi e^{-i \beta \sin (\phi+\psi)+i \alpha \psi} Q(\phi+\psi) \text {. }
$$

Using the expansion of the exponents over the cylindrical harmonics,

$$
e^{i \beta \sin \varphi}=\sum_{l} J_{l}(\beta) e^{i l \varphi}
$$

we find

$$
\begin{aligned}
& \int_{0}^{\infty} d \psi e^{-i \beta \sin (\phi+\psi)+i \alpha \psi} \\
& =\sum_{l} J_{l}(\beta) e^{i l \phi} \int_{0}^{\infty} e^{i(\alpha-l) \psi} d \psi=\sum_{l} \frac{J_{l}(\beta) e^{-i l \phi}}{i(l-\alpha)}
\end{aligned}
$$

and

$$
\begin{aligned}
& \int_{0}^{\infty} d \psi e^{-i \beta \sin (\phi+\psi)+i \alpha \psi} \cos (\phi+\psi) \\
& =\frac{i}{\beta} \frac{\partial}{\partial \phi} \int_{0}^{\infty} d \psi e^{-i \beta \sin (\phi+\psi)+i \alpha \psi}=\frac{1}{i \beta} \sum_{l} \frac{l J_{l}(\beta) e^{-i l \phi}}{l-\alpha} .
\end{aligned}
$$

Then Eq. (A6) transforms into

$$
\begin{aligned}
f_{1}(p, \phi)= & \frac{e^{i \beta \sin \phi}}{i \omega_{c}}\left(-\frac{\partial f_{0}}{\partial \varepsilon_{p}}\right) \\
& \times \sum_{l}\left[\frac{e \tilde{E}_{0} v}{\beta} l+\frac{n_{1}}{\tau} \frac{\partial \mu}{\partial n}\right] \frac{J_{l}(\beta)}{l-\alpha} e^{-i l \phi} .
\end{aligned}
$$

The conductivity tensor and the diffusion vector can be calculated using the standard definition of the first-order correction to the current density,

$$
j_{\alpha}^{(1)}=e \int \frac{d \mathbf{p}}{(2 \pi \hbar)^{2}} v_{\alpha} f_{1}(p, \phi)=\sigma_{\alpha \beta} \tilde{E}_{\beta}+e n_{1} R_{\alpha},
$$

where $\mathbf{v}(\phi)=v(\cos \phi, \sin \phi)$, and

$$
\begin{array}{r}
\sigma_{x x}=\frac{e^{2}}{\omega_{c}} \int_{0} \frac{d \mathbf{p}}{(2 \pi \hbar)^{2}} v^{2} \cos \phi e^{i \beta \sin \phi}\left(-\frac{\partial f_{0}}{\partial \varepsilon_{p}}\right) \\
\times \int_{0}^{\infty} d \psi e^{-i \beta \sin (\phi+\psi)+i \alpha \psi} \cos (\phi+\psi)
\end{array}
$$

and

$$
\begin{aligned}
R_{x}=\frac{1}{\omega_{c} \tau} \frac{\partial \mu}{\partial n} & \frac{d \mathbf{p}}{(2 \pi \hbar)^{2}} v \cos \phi e^{i \beta \sin \phi}\left(-\frac{\partial f_{0}}{\partial \varepsilon_{p}}\right) \times \\
& \times \int_{0}^{\infty} d \psi e^{-i \beta \sin (\phi+\psi)+i \alpha \psi}
\end{aligned}
$$

are the first $(x x)$ matrix element of the conductivity tensor and the $x$-component of the diffusion vector [51, 52, respectively. Taking integrals in A12 and in A13, we find the conductivity and the diffusion coefficient of a degenerate electron gas at zero temperature, Eqs. (4) and (5) in the main text.

\section{Appendix B: The second-order response and the AME current}

\section{a. Parabolic dispersion case}

Since we chose the SAW EM field to be directed along the $x$ axis, the AME current is given by the formula

$$
\begin{aligned}
j_{\alpha}= & -\frac{e^{2}}{2 \omega_{c}} \int \frac{d \mathbf{p}}{(2 \pi \hbar)^{2}} v_{\alpha}(\phi) \int_{0}^{\infty} d \psi e^{-\frac{\psi}{\omega_{c} \tau}} \\
& \times \operatorname{Re}\left\{\tilde{E}_{0}^{*} v \cos (\phi+\psi) \frac{\partial f_{1}(p, \phi+\psi)}{\partial \varepsilon_{p}}\right\} .
\end{aligned}
$$


Expressing the p-integrals via the integrals over the energy and angle, we perform partial integrations to find

$$
\begin{aligned}
\left(\begin{array}{l}
j_{x} \\
j_{y}
\end{array}\right)= & \operatorname{Re} \frac{e^{2} \tilde{E}_{0}^{*}}{\omega_{c}(2 \pi \hbar)^{2}} \int_{0}^{\infty} d \varepsilon_{\mathbf{p}} \int_{0}^{\infty} d \psi e^{-\frac{\psi}{\omega_{c} \tau}} \\
& \times \int_{0}^{2 \pi} d \phi\left(\begin{array}{c}
\cos \phi \\
\sin \phi
\end{array}\right) \cos (\phi+\psi) f_{1}(p, \phi+\psi)
\end{aligned}
$$

Substituting here the first-order electron distribution function A10, we come up with the $\phi$ and $\psi$-angle integrals,

$$
\begin{gathered}
\int_{0}^{2 \pi} d \phi \cos (\phi+\psi)\left(\begin{array}{c}
\cos \phi \\
\sin \phi
\end{array}\right) e^{i \beta_{F} \sin (\phi+\psi)} e^{-i l(\phi+\psi)} \\
=\frac{\pi}{\beta_{F}}\left(\begin{array}{c}
(l+1) J_{l+1}\left(\beta_{F}\right) e^{i \psi}+(l-1) J_{l-1}\left(\beta_{F}\right) e^{-i \psi} \\
i(l+1) J_{l+1}\left(\beta_{F}\right) e^{i \psi}-i(l-1) J_{l-1}\left(\beta_{F}\right) e^{-i \psi}
\end{array}\right), \\
\int_{0}^{\infty} d \psi e^{-\frac{\psi}{\omega_{c} \tau} \pm i \psi}=\omega_{c} \tau \frac{1 \pm i \omega_{c} \tau}{1+\left(\omega_{c} \tau\right)^{2}} .
\end{gathered}
$$

The integral over energy can be easily taken for a degenerate electrons gas, where $-\partial_{\varepsilon_{p}} f_{0}=\delta\left(\varepsilon_{p}-\mu\right)$. Summing up, we find Eq. 3 from the main text.

\section{b. Linear dispersion case}

Following similar steps as for the parabolic dispersion case, integrating by parts via energy, and taking into account that now the cyclotron frequency and the electron relaxation time depend on energy, we find

$$
\begin{aligned}
\left(\begin{array}{l}
j_{x} \\
j_{y}
\end{array}\right) & =\operatorname{Re} \frac{e^{2} \tilde{E}_{0}^{*}}{(2 \pi \hbar)^{2}} \\
& \times \int_{0}^{\infty} \frac{d \varepsilon_{\mathbf{p}}}{\omega_{c}(p)} \int_{0}^{\infty} d \psi e^{-\frac{\psi}{\omega_{c}(p) \tau_{2}(p)}}\left(1-\frac{\psi}{\omega_{c}(p) \tau_{2}(p)}\right) \\
& \times \int_{0}^{2 \pi} d \phi\left(\begin{array}{c}
\cos \phi \\
\sin \phi
\end{array}\right) \cos (\phi+\psi) f_{1}(p, \phi+\psi),
\end{aligned}
$$

[1] T. Ando, A. B. Fowler, and F. Stern, Electronic properties of two-dimensional systems, Rev. Mod. Phys. 54, 437 (1982).

[2] K. S. Novoselov, A. K. Geim, S. V. Morozov, D. Jiang, Y. Zhang, S. V. Dubonos, I. V. Grigorieva, and A. A. Firsov, Electric field effect in atomically thin carbon films, Science 306, 666 (2004).

[3] K. S. Novoselov, D. Jiang, F. Schedin, T. J. Booth, V. V. Khotkevich, S. V. Morozov, and A. K. Geim, Twodimensional atomic crystals, PNAS 102, 10451 (2005).

[4] Q. H. Wang, K. Kalantar-Zadeh, A. Kis, J. N. Cole- where

$$
\begin{aligned}
f_{1}(p, \phi)= & \frac{e^{i \beta_{p} \sin \phi}}{i \omega_{c}(p)}\left(-\frac{\partial f_{0}}{\partial \varepsilon_{p}}\right) \\
& \times \sum_{l}\left[\frac{e \tilde{E}_{0} v_{0}}{\beta} l+\frac{n_{1}}{\tau_{1}(p)} \frac{\partial \mu}{\partial n}\right] \frac{J_{l}\left(\beta_{p}\right)}{l-\alpha_{p}} e^{-i l \phi}
\end{aligned}
$$

The integration over $\phi$ is similar to the parabolic dispersion case, thus we find

$$
\begin{aligned}
& \int_{0}^{2 \pi} d \phi \cos (\phi+\psi)\left(\begin{array}{c}
\cos \phi \\
\sin \phi
\end{array}\right) e^{i \beta_{p} \sin (\phi+\psi)} e^{-i l(\phi+\psi)} \\
= & \frac{\pi}{\beta_{p}}\left(\begin{array}{c}
(l+1) J_{l+1}\left(\beta_{p}\right) e^{i \psi}+(l-1) J_{l-1}\left(\beta_{p}\right) e^{-i \psi} \\
i(l+1) J_{l+1}\left(\beta_{p}\right) e^{i \psi}-i(l-1) J_{l-1}\left(\beta_{p}\right) e^{-i \psi}
\end{array}\right),
\end{aligned}
$$

whereas for $\psi$-integrals, we use

$$
\begin{gathered}
\int_{0}^{\infty} d \psi e^{-\frac{\psi}{\omega_{c}(p) \tau_{2}(p)}} \pm i \psi\left(1-\frac{\psi}{\omega_{c}(p) \tau_{2}(p)}\right) \\
=\frac{\mp i \omega_{c}(p) \tau_{2}(p)}{\left[1 \mp i \omega_{c}(p) \tau_{2}(p)\right]^{2}} .
\end{gathered}
$$

The remaining integral over energy is much simpler in the case of the degenerate electron gas due to the relation $-\partial_{\varepsilon_{p}} f_{0}=\delta\left(\varepsilon_{p}-\mu\right)$, using which we find Eq. (7) in the main text. man, and M. S. Strano, Electronics and optoelectronics of two-dimensional transition metal dichalcogenides, Nature Nanotech. 7, 699 (2012).

[5] K. v. Klitzing, G. Dorda, and M. Pepper, New Method for High-Accuracy Determination of the Fine-Structure Constant Based on Quantized Hall Resistance, Phys. Rev. Lett. 45, 494 (1980).

[6] D. C. Tsui, H. L. Stormer, and A. C. Gossard, TwoDimensional Magnetotransport in the Extreme Quantum Limit, Phys. Rev. Lett. 48, 1559 (1982).

[7] R.B. Laughlin, Anomalous Quantum Hall Effect: An In- 
compressible Quantum Fluid with Fractionally Charged Excitations, Phys. Rev. Lett. 450, 1395 (1983).

[8] C. W. J. Beenakker and H. van Houten, Quantum transport in semiconductor nanostructures, Solid State Phys. 44, 1 (1991).

[9] G. Timp, A. M. Chang, J. E. Cunningham, T. Y. Chang, P. Mankiewich, R. Behringer, and R. E. Howard, Observation of the Aharonov-Bohm Effect for $\omega_{c} \tau>1$, Phys. Rev. Lett. 58, 2814 (1987).

[10] R. R. Gerhardts, D. Weiss, and K. V. Klitzing, Novel Magnetoresistance Oscillations in a Periodically Modulated Two-dimensional Electron Gas, Phys. Rev. Lett. 62, 1173 (1989).

[11] E. Brown, Bloch electrons in a uniform magnetic field, Phys. Rev. 133, A1038 (1964).

[12] J. Zak, Magnetic translation group, Phys. Rev. 134, A1602 (1964).

[13] D. R. Hofstadter, Energy levels and wave functions of Bloch electrons in rational and irrational magnetic fields, Phys. Rev. B 14, 2239 (1976).

[14] L. A. Ponomarenko, R. V. Gorbachev, G. L. Yu, D. D. Elias, R. Jalil, A. A. Patel, A. Mishchenko, A. S. Mayorov, C. R. Woods, J. R. Wallbank, M. MuchaKruczynski, B.A. Piot, M. Potemski, I. V. Grigorieva, K. S. Novoselov, F. Guinea, V. Falko, and A. K. Geim, Cloning of Dirac fermions in graphene superlattices, Nature (London) 497, 594 (2013).

[15] C. R. Dean, L. Wang, P. Maher, C. Forsythe, F. Ghahari, Y. Gao, J. Katoch, M. Ishigami, P. Moon, M. Koshino, T. Taniguchi, K. Watanabe, K. L. Shepard, J. Hone, and P. Kim, Hofstadter's butterfly and the fractal quantum Hall effect in moir superlattices, Nature (London) 497, 598 (2013).

[16] B. Hunt, J. D. Sanchez-Yamagishi, A. F. Young, M. Yankowitz, B. J. LeRoy, K. Watanabe, T. Taniguchi, P. Moon, M. Koshino, P. Jarillo-Herrero, and R. C. Ashoori, Massive Dirac Fermions and Hofstadter Butterfly in a van der Waals Heterostructure, Science 340, 427 (2013).

[17] R. Krishna Kumar, X. Chen, G. H. Auton, A. Mishchenko, D. A. Bandurin, S. V. Morozov, Y. Cao, E. Khestanova, M. Ben Shalom, A. V. Kretinin, K. S. Novoselov, L. Eaves, I. V. Grigorieva, L. A. Ponomarenko, V. I. Fal'ko, and A. K. Geim, High-temperature quantum oscillations caused by recurring Bloch states in graphene superlattices, Science 357, 181 (2017).

[18] V. L. Ginzburg, Usp. Fiz. Nauk. 69, 537 (1959).

[19] M. H. Cohen, M. J. Harrison, and W. A. Harrison, Phys. Rev. 117, 937 (1960).

[20] A. V. Chaplik and D. Heitmann, J . Phys. C: Solid State Phys. 183357 (1985).

[21] T. Ando, Phys. Rev. Lett. 36, 1383 (1976).

[22] E. G. Mohr and D. Heitmann, J. Phys. C: Solid State Phys. 15, L753 (1982).

[23] C. Campbell, Surface Acoustic Wave Devices for Mobile and Wireless Communications (Academic Press Inc, San Diego, CA, USA, 1998).

[24] R. H. Parmenter, The Acousto-Electric effect, Phys. Rev. 89, 990 (1953).

[25] C. C. W. Ruppel and T. A. Fjeldy, Advances in surface acoustic wave technology, systems and applications (World Scientific Publishing Co. Pte. Ltd., Singapore, 2001).

[26] S. H. Zhang and W. Xu, Absorption of surface acoustic waves by graphene, AIP Advances 1, 022146 (2011).
[27] V. Miseikis, J. E. Cunningham, K. Saeed, R. O'Rorke, and A. G. Davies, Acoustically induced current flow in graphene, Appl. Phys. Lett. 100, 133105 (2012).

[28] A. V. Kalameitsev, V. M. Kovalev, and I. G. Savenko, Valley Acoustoelectric Effect, Phys. Rev. Lett. 122, 256801 (2019).

[29] V. Parente, A. Tagliacozzo, F. von Oppen, and F. Guinea, Electron-phonon interaction on the surface of a three-dimensional topological insulator, Phys. Rev. B 88, 075432 (2013).

[30] L. L. Li and W. Xu, Absorption of surface acoustic waves by topological insulator thin films, Appl. Phys. Lett. 105, 063503 (2014).

[31] E. G. Batyev, V. M. Kovalev, A. V. Chaplik, Response of a Bose-Einstein condensate of dipole excitons to static and dynamic perturbations, JETP Lett. 99(9), 540 (2014).

[32] V. M. Kovalev and A. V. Chaplik, Effect of exciton dragging by a surface acoustic wave, JETP Lett. 101(3), 177 (2015).

[33] V. M. Kovalev and A. V. Chaplik, Acousto-exciton interaction in a gas of $2 \mathrm{D}$ indirect dipolar excitons in the presence of disorder, JETP 122(3), 499 (2016).

[34] A. Wixforth, J. Scriba, M. Wassermeier, J. P. Kotthaus, G. Weimann, and W. Schlapp, Surface acoustic waves on GaAs $/ \mathrm{Al}_{x} \mathrm{Ga}_{1-x}$ As heterostructures, Phys. Rev. B 40, 7874 (1989).

[35] R. L. Willett, M. A. Paalanen, R.R. Ruel, K.W. West, L.N. Pfeiffer, and D.J. Bishop, Anomalous Sound Propagation at $\nu=1 / 2$ in a $2 \mathrm{D}$ Electron Gas: Observation of a Spontaneously Broken Translational Symmetry?, Phys. Rev. Lett. 65, 112 (1990).

[36] D. J. Kreft and R. H. Blick, Surface Acoustic Waves and Nano-Electromechanical Systems, Acoustic WavesFrom Microdevices to Helioseismology, edited by M.G. Beghi (InTech, 2011).

[37] D. J. Kreft, L. G. Mourokh, H. Shin, M. Bichler, W. Wegscheider, and R. H. Blick, Giant acoustoelectric current in suspended quantum point contacts, Phys. Rev. B 94, 235305 (2016).

[38] Y. Levinson, O. Entin-Wohlmann, A. D. Mirlin, and P. Wolfle, Weiss oscillations in surface-acoustic-wave propagation, Phys. Rev. B 58, 7113 (1998).

[39] C. Eckl, Yu. A. Kosevich, and A. P. Mayer, Surface acoustic waves and magnetotransport in an embedded modulated two-dimensional electron gas, Phys. Rev. B 61, 16708 (2000).

[40] J. P. Robinson and V. I. Falko, Commensurability oscillations in the surface-acoustic-wave-induced acoustoelectric effect in a two-dimensional electron gas, Phys. Rev. B 71, 241301(R) (2005).

[41] M. M. Glazov and S. D. Ganichev, High frequency electric field induced nonlinear effects in graphene, Phys. Rep. 535, 101 (2014).

[42] Zh. Sun, D. N. Basov, and M. M. Fogler, Third-order optical conductivity of an electron fluid, Phys. Rev. B 97, 075432 (2018).

[43] V. M. Kovalev and I. G. Savenko, Photogalvanic currents in dynamically gapped transition metal dichalcogenide monolayers, Phys. Rev. B 99, 075405 (2019).

[44] A. V. Nalitov, L. E. Golub, and E. L. Ivchenko, Ratchet effects in two-dimensional systems with a lateral periodic potential, Phys. Rev. B 86, 115301 (2012).

[45] A. M. Witowski, M. Orlita, R. Stȩpniewski, A. 
Wysmołek, J. M. Baranowski, W. Strupiński, C. Faugeras, G. Martinez, and M. Potemski, Quasiclassical cyclotron resonance of Dirac fermions in highly doped graphene, Phys. Rev. B 82, 165305 (2010).

[46] M. Orlita, I. Crassee, C. Faugeras, A. B. Kuzmenko, F. Fromm, M. Ostler, Th. Seyller, G. Martinez, M. Polini, and M. Potemski, Classical to quantum crossover of the cyclotron resonance in graphene: A study of the strength of intraband absorption, New J. Phys. 14, 095008 (2012).

[47] J.-H. Chen, C. Jang, S. Xiao, M. Ishigami, and M. S. Fuhrer, Intrinsic and extrinsic performance limits of graphene devices on $\mathrm{SiO}_{2}$, Nature Nanotech. 3, 206 (2008).

[48] A. Akturka and N. Goldsman, Electron transport and full-band electron-phonon interactions in graphene, J. Appl. Phys. 103, 053702 (2008).
[49] J. Karch, P. Olbrich, M. Schmalzbauer, C. Brinsteiner, U. Wurstbauer,M. M. Glazov, S. A. Tarasenko, E. L. Ivchenko, D. Weiss, J. Eroms, and S. D. Ganichev, Photon helicity driven electric currents in graphene, Proceedings of the 35th International Conference on Infrared, Millimeter, and Terahertz Waves (2010).

[50] E. M. Lifshitz and L. P. Pitaevskii, Landau and Lifshitz Course of Theoretical Physics. Volume X. Physical Kinetics. (Translated from Nauka, Moscow, 1979). We used pages $220-222$.

[51] M. V. Krasheninnikov and A. V. Chaplik, Plasmaacoustic waves on the surface of a piezoelectric crystal, JETP 48, 960 (1978).

[52] C. Kittel, Quantum theory of solid states (Wiley, New York, 2004). 